\title{
Propionyl-CoA Carboxylase Deficiency in a Patient with Biotin-responsive 3-Methylcrotonylglycinuria
}

\author{
LAWRENCE SWEETMAN, ${ }^{(31)}$ SETH P. BATES, DAVID HULL, AND WILLIAM L. NYHAN \\ Department of Pediatrics, University of California, San Diego, La Jolla, California, USA, and Department of Child \\ Health, The University of Nottingham, Nottingham, England
}

\section{Summary}

The abnormal metabolites 3-hydroxypropionic acid (1.6-4.0 $\mathrm{mg} /$ day) and methylcitric acid (3.7-5.8 mg/day) were identified and quantitated in the urine of a patient in whom biotin-responsive 3-methylcrotonylglycinuria and deficiency of 3-methylcrotonyl-CoA carboxylase had previously been documented. The level of excretion of these metabolites was in the lower range of those found in patients with propionic acidemia in whom there is a deficiency of propionyl-CoA carboxylase. The activity of this enzyme in fibroblasts derived from the patient and grown in media low in biotin was $4 \%$ of normal. This is in the range of patients with propionyl-CoA carboxylase deficiency. Documented deficiency in this patient of two carboxylases, both of which contain biotin, suggests that the primary defect is in the metabolism of biotin.

\section{Speculation}

The deficiency of two mitochondrial carboxylases in a patient suggests the presence of a fundamental defect in either the transport of biotin or in the holocarboxylase synthetase that attaches biotin covalently to both carboxylases.

3-Methylcrotonylglycinuria is a rare inborn error of leucine metabolism which is characterized by excessive excretion of 3methylcrotonylglycine and 3-hydroxyisovaleric acid. The second patient reported differed from the first $(10,20)$ in that he was severely ketoacidotic, and he responded both clinically and biochemically to biotin (14). Biotin is a cofactor of 3-methylcrotonyl-CoA carboxylase. Deficiency of 3-methylcrotonyl-CoA carboxylase was demonstrated in leukocytes from this patient (15). A third patient reported (12) was not ketotic but responded to biotin.

The second patient appeared to be unique in the occurrence of severe ketoacidosis and the fact that he was found to excrete tiglylglycine $(13,14)$. Tiglylglycine is a metabolite of isoleucine. It is excreted by patients with propionic acidemia (19). Therefore, we analyzed the urine of this patient in the search for the abnormal metabolites found in patients with propionic acidemia (3). It is the purpose of this report to describe the identification and quantitation of methylcitric acid and 3-hydroxypropionic acid in the urine. Enzyme assay of cultured fibroblasts demonstrated a deficiency of propionyl-CoA carboxylase. A preliminary report of these results has appeared in abstract form (23). At the same time, Chalmers et al. (8) reported elevated amounts of propionic and 3-methylcrotonic acid in urine from this patient. They also identified 3-hydroxypropionic acid and made a tentative identification of methylcitric acid in the urine (9).

\section{MATERIALS AND METHODS}

\section{SUBJECT}

At the times the urine samples were collected for analysis, the patient $J R$ was 21 or 23 months of age. His weight was $11 \mathrm{~kg}$ and his height $81.6 \mathrm{~cm}$. His level of development was normal for his age. He was receiving $0.5 \mathrm{mg} /$ day biotin, and a protein intake of about $3.2-3.6 \mathrm{~g} / \mathrm{kg}$.day. The patient was in excellent condition and not ketotic or acidotic. Specimens were obtained with informed consent.

\section{ANALYSIS OF URINE}

Urinary organic acids were analyzed quantitatively by liquid partition chromatography on $\mathrm{H}_{2} \mathrm{SO}_{4}$-hydrated silicic acid eluted with a gradient of $t$-amyl alcohol in chloroform. The eluted acids were continuously titrated by mixing with sodium $o$-nitrophenol $(16,21)$ and the titration of the indicator was recorded spectrophotometrically by the increase in absorbance at $350 \mathrm{~nm}$. Some analyses were performed at twice the usual flow rate to shorten the time of analysis to $3.5 \mathrm{hr}$. Equivalents of acid were calculated from peak areas relative to peak areas of standards. Fractions obtained from liquid partition chromatography were analyzed further by gas liquid chromatography (GLC) and mass spectrometry (GCMS) of the trimethylsilyl (TMS) derivatives on Dexsil 300 using an HP 402 gas chromatograph and an LKB 9000 gas chromatograph-mass spectrometer as previously described (21). Retention times on GLC were expressed as methylene unit (MU) values by co-injection of even-numbered alkanes. Acids coeluted on the liquid partition chromatography system were quantitated by GLC.

\section{ENZYME ASSAY}

Fibroblasts derived from a biopsy of the skin were grown in culture in Eagle's minimal essential medium (MEM) with $10 \%$ fetal calf serum. Cells were harvested by trypsinization and lysed by freezing and thawing three times in $0.05 \mathrm{M}$ Tris- $\mathrm{HCl}$ at $\mathrm{pH}$ 8.0. Propionyl-CoA carboxylase was assayed by the incubation of $5 \times 10^{6}$ cells (about $1 \mathrm{mg}$ protein) in $1.5 \mathrm{ml}$ at $30^{\circ}$ for $30 \mathrm{~min}$ in the presence of $\mathrm{NaH}^{14} \mathrm{CO}_{3}$ and measurement of the fixation of $\mathrm{CO}_{2}$ to propionyl-CoA as described by Tietz and Ochoa (25), except for the addition of $50 \mathrm{mM} \mathrm{KCl}$ to the assay (11). Unreacted $\mathrm{H}_{2}{ }^{14} \mathrm{CO}_{33}$ was removed by acidification with trichloroacetic acid and drying overnight under a heat lamp. Nonvolatile ${ }^{14} \mathrm{C}$ incorporated in the presence of propionyl-CoA minus a cell blank without propionyl-CoA represented product. Protein was determined by the biuret method (17).

\section{RESULTS}

Typical liquid partition chromatograms for a normal subject and patient $J R$ are shown in Figure 1. The amounts of urine 
studied contained $1.0 \mathrm{mg}$ creatinine. Retention times of acid peaks are expressed as $R_{F}$ values relative to citric acid which is equated to 100.0 . The identities of the acids in all peaks were confirmed by comparison of MU values on GLC and the mass spectra of TMS derivatives of authentic standards. The major normal peaks in both urines were hippuric acid at $R_{F} 8.5-$ 10.6 , citric acid at $R_{F} 100.00$, and isocitric acid at $R_{F} 102.9$ 103.0. In the urine of $J R$ the major elevated peak at $R_{F} 16.1$ was 3-hydroxyisovaleric acid. The small peak at $R_{F} 17.6$ in the normal was $p$-hydroxyphenylacetic acid, which also contributed to the $R_{F} 16.1$ peak in patient $J R$. The peak at $R_{F} 12.2$ in $J R$ was 3-methylcrotonylglycine. The peak at 14.4 in the normal was acetic acid. The peak at $R_{F} 61.8-63.9$ in the normal contained trans-aconitic acid and pyrrolidone-2-carboxylic acid. As shown in Figure 2, the peak at $R_{F} 62.5$ in $J R$ also contained 3-hydroxypropionic acid. The double peaks at $\mathrm{R}_{\mathrm{F}} 83.0$ and 85.4 in $J R$ were the erythro and threo isomers of methylcitric acid. Figure 3 shows that the mass spectra of the TMS derivative of the $R_{F} 85.4$ peak was identical to that of the tetra-TMS derivative of authentic methylcitric acid. The diastereoisomer at $R_{F} 83.0$ had an identical mass spectra. No methylcitric acid was detected in the normal subjects. Standard homocitric acid (3-carboxyl-3-hydroxyadipic acid) had an $R_{F}$ of 86.7 on liquid partition chromatography and the mass spectrum of the TMS derivative was very similar to that of methylcitric acid. However, the MU value for the TMS derivative of homocitric acid on GLC was 19.20, which is quite different from those of the diasterioisomers of standard methylcitric acid $\left(R_{F} 83.3, M U 18.19 ; R_{F} 85.4\right.$, MU 18.28). No homocitric acid was detected in urine of $J R$, patients with propionic acidemia, or normal subjects. An unknown compound with a MU value of 16.38 and an apparent TMS molecular weight of 438 was found at $R_{F} 83.0$ in the normal subject and the patient. Although the patient excreted 3-hydroxyisovaleric acid and 3-methylcrotonylglycine as expected, no tiglylglycine $\left(R_{F}\right.$ $14.6)$ was detected. The urine of $J R$ contained no propionylglycine $\left(R_{F} 45.5\right)$ or methylmalonic acid $\left(R_{F} 41.2\right)$.

The quantities of abnormal metabolites excreted by $J R$ at 21 and 23 months are shown in Table 1 . The excretion of $3-$ methylcrotonylglycine was $17-48 \mathrm{mg} /$ day and that of 3-hydroxysiovaleric acid was $66-83 \mathrm{mg} / \mathrm{day}$. The levels of excretion of 3- hydroxypropionic acid were $1.6-4.0 \mathrm{mg} /$ day and that of methylcitric acid 3.7-5.8 mg/day.

These findings, along with evidence for decreased oxidation of $\left[{ }^{14} \mathrm{C}\right]$ propionic acid to ${ }^{14} \mathrm{CO}_{2}$ in fibroblasts derived from $J R(2)$, strongly suggested a deficiency of propionyl-CoA carboxylase. Data on propionyl-CoA carboxylase activity in fibroblasts are shown in Table 2 . In $J R$ the activity of this enzyme was $4 \%$ of normal. This value was within the range of activity of cells from a series of four patients with propionic acidemia, in whom pro-

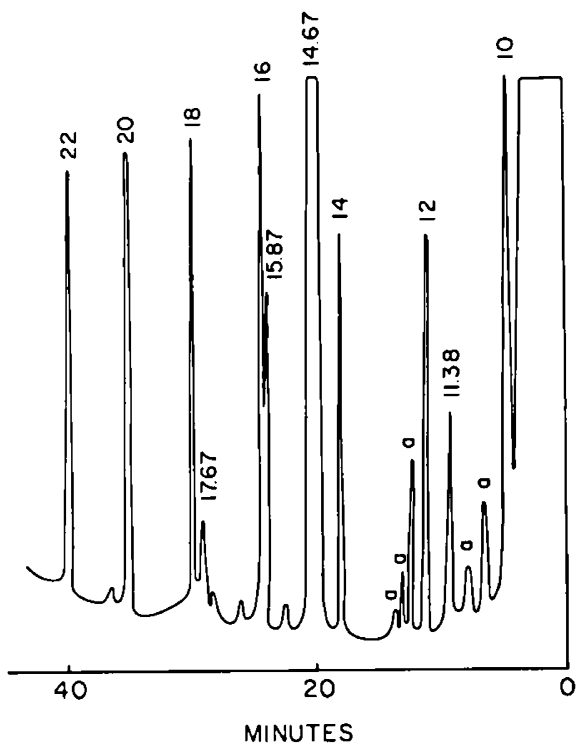

Fig. 2. Gas liquid chromatogram of trimethylsilyl derivatives of the $R_{F}$ 62.5 peak from liquid partition chromatography of urine of patient $J R$. The numbers above the peaks are methylene unit values. The major peaks are: 11.38 3-hydroxypropionic acid; $14.67 \mathrm{o}$-nitrophenol; 15.87 pyrrolidone-2-carboxylic acid; and 17.67 trans-aconitic acid. Solvent and reagent artifacts are indicated by $a$. The column was Dexsil $300(4 \mathrm{~mm} \times$ $6 \mathrm{foot}$ ) with a He carrier flow of $50 \mathrm{ml} / \mathrm{min}$, an initial temperature of $60^{\circ}$, programmed at $4 \% \mathrm{~min}$.
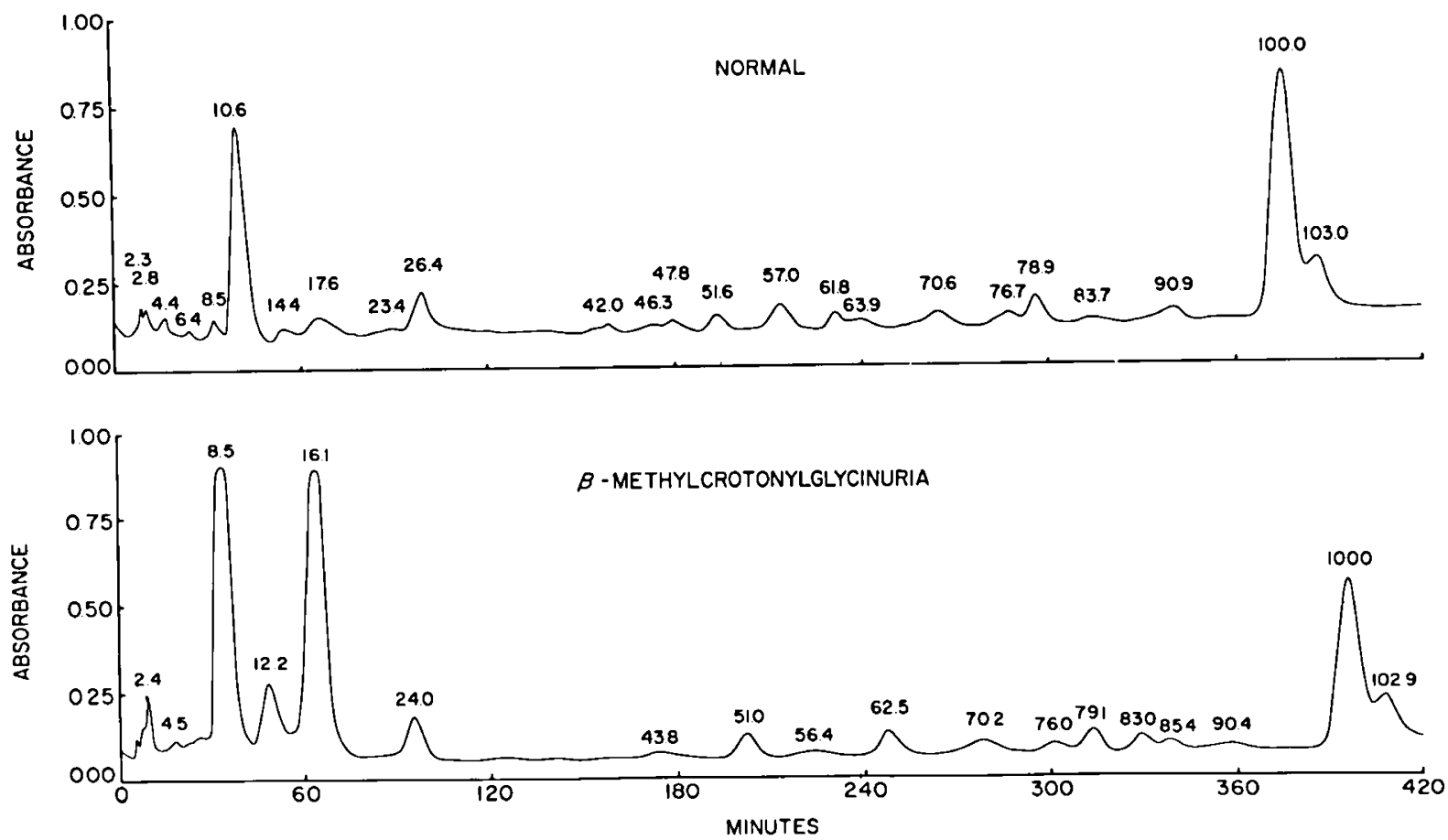

Fig. 1. Liquid partition chromatograms of organic acids in amounts of urine containing $1 \mathrm{mg}$ creatinine. The absorbance is proportional to the amount of acid present. The numbers above the peaks are $R_{F}$ values relative to citric acid equated to 100.0. 
pionyl-CoA carboxylase was defective. These assays were done with cells grown in MEM which contains no biotin except that contributed by the $10 \%$ fetal calf serum. Gompertz et al. (15) had previously shown a partial deficiency of 3-methylcrotonylCoA carboxylase in leukocytes from this patient. Furthermore, we have demonstrated deficiency of 3-methylcrotonyl-CoA carboxylase in fibroblasts (28). Thus, the patient had deficient activity of two biotin-containing carboxylases.

\section{DISCUSSION}

This boy $(J R)$ was the patient originally reported by Gompertz, Hull, and colleagues (14) under the designation of biotinresponsive $\beta$-methylcrotonylglycinuria. It is important to distinguish this patient from the patient with biotin-responsive propionic acidemia also reported by Hull, Gompertz, and colleagues (6). $J R$ had a remarkable therapeutic response to biotin. Nevertheless, the levels of excretion of 3-methylcrotonylglycine and 3-hydroxyisovaleric acid found in this study were appreciable while he was receiving $0.5 \mathrm{mg}$ biotin/day. The amounts of $3-$ methylcrotonylglycine $(17-48 \mathrm{mg} /$ day $)$ were less than those observed at 5 months of age when he was acutely ill and ketotic. At that time, excretion of 3-methylcrotonylglycine was $50-250 \mathrm{mg} /$ day, whereas that of 3-hydroxyisovaleric acid was consistently less (14). Following treatment at that time with $10 \mathrm{mg}$ biotin/ day, these metabolites were not detectable (14). As the patient

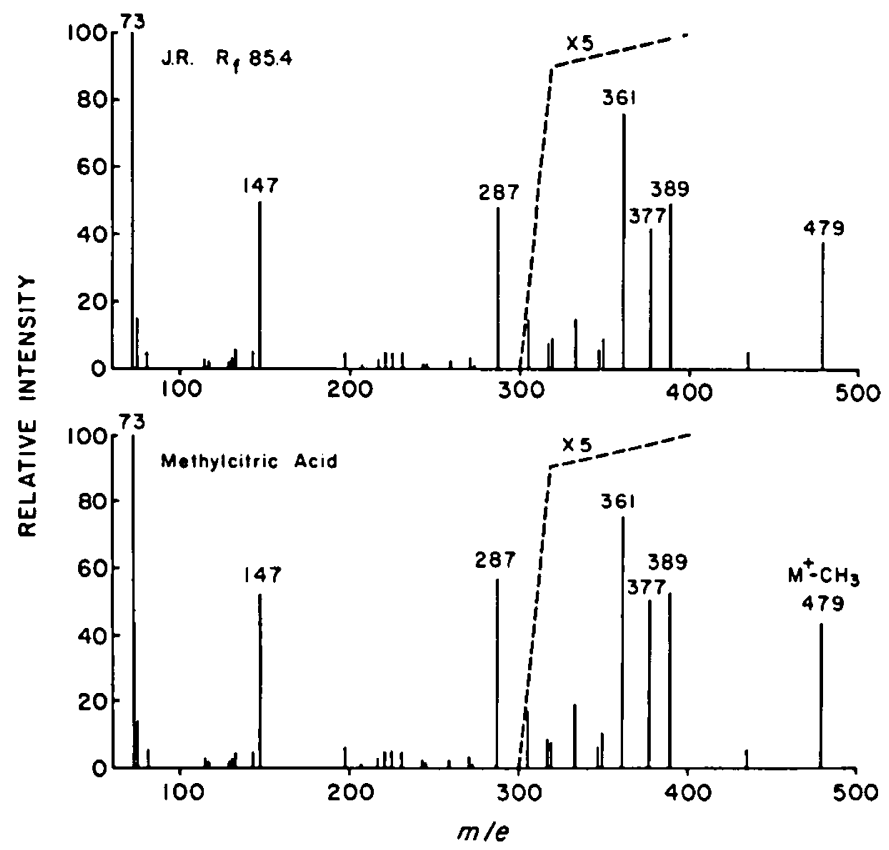

Fig. 3. Mass spectra of trimethylsilyl derivatives of authentic methylcitric acid and methylcitric acid from $R_{F} 85.4$ peak of patient $J R$. The spectra were obtained with an ionizing voltage of $70 \mathrm{eV}$. Relative intensities of ions are plotted against mass/charge $(\mathrm{m} / \mathrm{e})$ ratios. Only the fragments containing the major isotope of silicon are shown. grew, the regimen of biotin treatment was sufficient to prevent illness but not sufficient to prevent the excretion of the abnormal products of leucine metabolism. It is of interest that considerably more 3-hydroxyisovaleric acid than 3-methylcrotonylglycine was excreted, the reverse of the situation when the patient was acutely ill.

The levels of excretion of 3-hydroxypropionic acid and methylcitric acid were in the lower range of those found in patients with propionic acidemia due to propionyl-CoA carboxylase deficiency $(22,26)$. This is also consistent with an effect of biotin treatment. When the patient was acutely ill, he had excreted $100-150 \mathrm{mg} /$ day of tiglylglycine (14). The absence of tiglylglycine in urine at the time of this study was consistent with the finding of relatively low levels of excretion of 3-hydroxypropionic acid and methylcitric acid. Patients with propionic acidemia excrete tiglylglycine only when acutely ill or under conditions of poor therapeutic control (19), conditions that also cause high levels of excretion of 3-hydroxypropionic acid and methylcitric acid. Propionylglycine has been found in the urine of patients with propionic acidemia (18). No propionylglycine could be detected in the urine of $J R$. The excretion of glycine by $J R$ was normal $(0.17 \mathrm{mg} / \mathrm{mg}$ creatinine $)$. In our experience, the excretion of methylcitric acid in appreciable amounts is the single most reliable index of a defect in the metabolism of propionic acid. The definitive chemical identification of this compound and the precise quantitation of its level of excretion are the contributions of this study and play a significant role in the development of the current level of understanding of this important patient. The metabolic patterns displayed in the urinary organic acids strongly suggested that there was a deficiency of propionyl-CoA carboxylase activity $(4,5)$ as well as 3 -methylcrotonyl-CoA carboxylase activity.

Chalmers et al. (9) have reported the identification by GCMS of 3-hydroxypropionic acid in the urine of $J R$. These authors also reported the tentative identification of methylcitric acid, although they could not exclude the possibility that the compound was homocitric acid. We have found that the urine of this patient contains no detectable homocitric acid nor does the urine of patients with propionyl-CoA carboxylase deficiency. The urine specimen analyzed by Chalmers $e t$ al. contained very high levels of 3-hydroxybutyric acid, indicating that the patient was markedly ketotic and presumably not receiving biotin. The quantities of metabolites excreted were expressed as gas chromatographic peak areas relative to peak areas of creatinine. Assuming similar response factors, the excretion of 3-hydroxypropionic acid was about half that of 3-hydroxyisovaleric acid and the excretion of methylcitric acid was much greater than that of citric acid. These

Table 2. Activity of propionyl-CoA carboxylase in fibroblasts

\begin{tabular}{lc}
\hline & $\mathrm{nmol} / \mathrm{mg}$ protein $\cdot \mathrm{hr}^{1}$ \\
\hline 3-Methylcrotonylglycinuria $(J R)$ & 1.1 \\
Propionic acidemia $(n=4)$ & $0.56 \pm 0.34$ \\
Normal $(n=5)$ & $26.58 \pm 10.04$ \\
\hline
\end{tabular}

1 Value for each subject represented the mean of two or more determinations. The means \pm SD were given for patients with propionic acidemia and for control individuals.

Table 1. Urinary excretion of organic acids by patient

\begin{tabular}{|c|c|c|c|c|c|}
\hline \multirow[b]{2}{*}{ Organic acid } & \multirow[b]{2}{*}{$\mathbf{R}_{\mathbf{F}}$} & \multicolumn{4}{|c|}{$\mu \mathrm{mol} / \mathrm{mg}$ creatinine } \\
\hline & & \multicolumn{2}{|c|}{$21 \mathrm{mo}^{1}$} & \multicolumn{2}{|c|}{$23 \mathrm{mo}^{1}$} \\
\hline 3-Methylcrotonylglycine & 12.2 & 1.79 & 2.86 & 1.06 & 2.52 \\
\hline 3-Hydroxyisovaleric acid & 16.1 & 8.85 & 9.59 & 5.62 & 5.82 \\
\hline 3-Hydroxypropionic acid & 62.5 & 0.27 & 0.36 & 0.42 & 0.26 \\
\hline Methylcitric acid & $83.0,85.4$ & 0.28 & 0.31 & 0.27 & 0.20 \\
\hline 24-Hr urine volume $(\mathrm{ml})$ & & 182 & 102 & 110 & 253 \\
\hline Creatinine $(\mathrm{mg} / \mathrm{ml})$ & & 0.35 & 0.60 & 0.95 & 0.48 \\
\hline
\end{tabular}

\footnotetext{
${ }^{1}$ Two consecutive $24-\mathrm{hr}$ urines were analyzed at each age.
} 
observations suggest that the activity of propionyl-CoA carboxylase in vivo was quite low at the time the urine was studied.

The excretion of all of the abnormal metabolites (3-hydroxyisovaleric acid, 3-methylcrotonylglycine, 3-hydroxypropionic acid, and methylcitric acid) suggests the possibility of an approach to the rapid prenatal diagnosis of this disorder. We have recently found that methylcitric acid is detectable in the amniotic fluid bathing a fetus with propionyl-CoA carboxylase deficiency (24). The use of organic acid analysis and gas chromatographymass spectrometry provides an approach to prenatal diagnosis in which direct chemical analysis of the fluid can save the 3-6-week delay required for the growth in culture of cells from amniotic fluid.

The severe ketoacidosis seen in $J R$ before biotin therapy is a characteristic symptom of propionic acidemia (3). It was not seen in the other patients reported with 3-methylcrotonylglycinuria $(10,12,20)$. Thus, it is likely that this aspect of the clinical picture is due not to deficiency of 3-methylcrotonyl-CoA carboxylase but rather to the deficiency of propionyl-CoA carboxylase.

It is now apparent that this patient has a biotin-responsive deficiency of propionyl-CoA carboxylase as well as 3-methylcrotonyl-CoA carboxylase. Bartlett and Gompertz (7) and Weyler et al. (27) have now demonstrated parallel deficiencies of both enzymes in fibroblasts derived from $J R$. Evidence for the deficiency of two biotin-containing acyl-CoA carboxylases in this patient is consistent with evidence for a biotin-responsive decrease in the excretion of the abnormal metabolities. Together, they strongly suggest that the primary defect is in the metabolism of biotin. Separate primary defects in two carboxylases would be unlikely. Normal activity of both carboxylases has been shown in fibroblasts of this patient when cultured in media containing high concentrations of biotin $(7,27)$. Abnormality in the activity of these two enzymes at low concentrations of biotin could represent either a defect in the transport of biotin or a defect in the enzyme holocarboxylase synthetase (1) that attaches biotin covalently to the inactive apocarboxylases to form the enzymatically active holocarboxylases. Preliminary experiments suggest that a defect in holocarboxylase synthetase is more likely.

\section{CONCLUSION}

A patient initially described as having biotin-responsive 3methylcrotonylglycinuria was shown to excrete elevated amounts of 3-hydroxypropionic acid and methylcitric acid, abnormal metabolites found in propionic acidemia, while receiving $0.5 \mathrm{mg}$ biotin/day. This evidence for in vivo deficiency of propionyl-CoA carboxylase activity was confirmed by finding a marked deficiency of propionyl-CoA carboxylase activity in fibroblasts cultured in medium containing low concentrations of biotin.

\section{REFERENCES AND NOTES}

1. Achuta-Murthy, P. N., and Mistry, S. P.: Synthesis of biotin-dependent carboxylases from their apoproteins and biotin. J. Sci. Ind. Res., 31: 554 (1972).

2. Ando, T., and Nyhan, W. L.: Unpublished observations.

3. Ando, T., and Nyhan, W. L.: Propionic acidemia and the ketotic hyperglycinemia syndrome. In: W. L. Nyhan: Heritable Disorders of Amino Acid Metabolism, p. 37 (Wiley \& Sons, New York, 1974).

4. Ando, T., Rasmussen, K., Nyhan, W. L., and Hull, D.: 3-Hydroxypropionate: significance of $\beta$-oxidation of propionate in patients with propionic acidemia and methylmalonic acidemia. Proc. Natl. Acad. Sci. U. S. A., 69: 2807 (1972).

5. Ando, T., Rasmussen, K., Wright, J. M., and Nyhan, W. L.: Isolation and identification of methylcitrate, a major metabolic product of propionate in patients with propionic acidemia. J. Biol. Chem., 247: 2200 (1972).

6. Barnes, N. D., Hull, D., Balgobin, L., and Gompertz, D.: Biotin-responsive propionic acidaemia. Lancet, ii: 224 (1970).

7. Bartlett, K., and Gompertz, D.: Combined carboxylase defect: Biotin-responsiveness in cultured fibroblasts. Lancet, ii: 804 (1976).

8. Chalmers, R. A., Bickle, S., and Watts, R. W. E.: A method for the determinations of volatile organic acids in aqueous solutions and urine, and the results obtained in propionic acidaema, $\beta$-methylcrotonylglycinuria and methylmalonic aciduria. Clin. Chim. Acta, 52: 31 (1974).

9. Chalmers, R. A., Lawson, A. M., and Watts, R. W. E.: Studies on the urinary acidic metabolites excreted by patients with $\beta$-methylcrotonylglycinuria, propionic acidaemia and methylmalonic acidaemia, using gas-liquid chromatography and mass spectrometry. Clin. Chim. Acta, 52: 43 (1974).

10. Eldjarn, L., Jellum, E., Stokke, O., Pande, H., and Waaler, P. E.: $\beta$ Hydroxyisovaleric aciduria and $\beta$-methylcrotonylglycinuria: a new inborn error of metabolism. Lancet, ii: 521 (1970).

11. Giorgio, A. J., and Whitaker, T. R.: Some properties of propionyl-CoA carboxylase partially purified from human liver. Biochem. Med., 7: 473 (1973).

12. Gompertz, D., Bartlett, K., Blair, D., and Stern, C. M. M.: Child with a defect in leucine metabolism associated with $\beta$-hydroxyisovaleric aciduria and $\beta$-methylcrotonylglycinuria. Arch. Dis. Childhood, 48: 975 (1973).

13. Gompertz, D., and Draffan, G. H.: The identification of tiglylglycine in the urine of a child with $\beta$-methylcrotonylglycinuria. Clin. Chim. Acta, 37: 405 (1972).

14. Gompertz, D., Draffan, G. H., Watts, J. L., and Hull, D.: Biotin-responsive $\beta$-methylcrotonylglycinuria. Lancet, $i i: 22$ (1971).

15. Gompertz, D., Goodey, P. A., and Bartlett, K.: Evidence for the enzymic defect in $\beta$-methylcrotonylglycinuria. FEBS Lett., 32: 13 (1973).

16. Kesner, L., and Muntwyler, E.: Automatic determination of weak organic acids by partition column chromatography and indicator titration. Anal. Chem. 38: 1164 (1966).

17. Layne, E.: Spectrophotometric and turbidimetric methods for measuring proteins. In: S. D. Colowick and N. O. Kaplan: Methods in Enzymology, Vol. III, p. 447 (Academic Press, New York, 1957).

18. Rasmussen, K., Ando, T., Nyhan, W. L., Hull, D., Cottom, D., Donnell, G., Wadlington, W., and Kilroy, A. W.: Excretion of propionylglycine in propionic acidemia. Clin. Sci., 42: 665 (1972).

19. Rasmussen, K., Ando, T., Nyhan, W. L., Hull, D., Cottom, D., Kilroy, A W., and Wadlington, W.: Excretion of tiglylglycine in propionic acidemia. J. Pediat. 81: 970 (1972).

20. Stokke, O., Eldjarn, L., Jellum, E., Pande, H., and Waaler, P. E.: $\beta$ methylcrotonyl-CoA carboxylase deficiency: A new metabolic error in leucine degradation. Pediatrics, 49: 726 (1972)

21. Sweetman, L.: Liquid partition chromatography and gas chromatographymass spectrometry in identification of acid metabolities of amino acids. In: W. L. Nyhan: Heritable Disorders of Amino Acid Metabolism, p. 730 (Wiley \& Sons, New York, 1974).

22. Sweetman, L., and Nyhan, W. L.: Unpublished observations.

23. Sweetman, L., and Nyhan, W. L.: Propionyl-CoA carboxylase deficiency in $\beta$ methylcrotonylglycinuria [Abstr.]. Clin. Res., 22: 237A (1974).

24. Sweetman, L., Weyler, W., Shafai, T., Young, P. E., and Nyhan, W. L.: Prenatal diagnosis of propionic acidemia by organic acid analysis. [Abstr.] Clin. Res., 24: 295A (1976).

25. Tietz, A., and Ochoa, S.: Propionyl-CoA carboxylase from pig heart. In: S. P. Colowick and N. O. Kaplan: Methods in Enzymology, Vol. V, p. 570 (Academic Press, New York, 1962).

26. Wadlington, W. B., Kilroy, A., Ando, T., Sweetman, L., and Nyhan, W. L.: Hyperglycinemia and propionyl-CoA carboxylase deficiency and episodic severe illness without consistent ketosis. J. Pediat., 86: 707 (1975).

27. Weyler, W., Sweetman, L., Maggio, D. C., and Nyhan, W. L.: Deficiency of propionyl-CoA carboxylase and methylcrotonyl-CoA carboxylase in a patient with methylcrotonylglycinuria. Clin. Chim. Acta, 76: 321 (1977).

28. Weyler, W., Sweetman, L., and Nyhan, W. L.: Demonstration of in vitro responsiveness to biotin in fibroblasts of a patient with 3-methylcrotonylglycinuria and propionic acidemia. [Abstr.] Clin. Res., 24: 295A (1976).

29. The authors thank Mrs. Janette Holm for her expert technical assistance in the GCMS analyses.

30. This study was supported by United States Public Health Service Grant HD04608 from the National Institute of Child Health and Human Development, and National Institutes of Health Research Resource Grant RR00708 from the Biotechnology Resource Branch.

31. Requests for reprints should be addressed to: L. Sweetman, Ph.D., Department of Pediatrics M-009, U.C.S.D., La Jolla, Calif. 92093 (USA).

32. Received for publication November 24, 1976.

33. Accepted for publication April 5, 1977. 